\title{
Optical Limiting Behavior of Acid Blue 29 under a Low Power CW He-Ne Laser Irradiation
}

\author{
M.D. Zidan*, A.A. Mani, A.W. Allaf, Z. Ajuj and A. Allahham \\ Department of Physics, Atomic Energy Commission, P.O. Box 6091, Damascus, Syria
}

(Received January 30, 2009; in final form March 23, 2009)

\begin{abstract}
Optical limiting performances of acid blue 29 are investigated under irradiation by a $35 \mathrm{~mW}$ continuous wave $\mathrm{He}-\mathrm{Ne}$ laser. The optical limiting behavior is investigated by using transmission measurement through samples with different concentrations. Our results show that the optical limiting efficiency is concentrations dependence. Induced self-diffraction patterns are observed at relatively high intensities starting from $15 \mathrm{~mW}$.
\end{abstract}

PACS numbers: 42.65.Ky, 42.70.Nq, 42.70.jk, 84.30.Qi

\section{Introduction}

With the development of laser technology, much interest in the development of optical limiting materials has been made among large number of researches working on materials. Devices for human eyes protection and solid-state sensors from intense laser beams are sought [1-3]. Previous researches on optical limiting materials were focused on optical nonlinear organic $[4,5]$ and semiconductor materials [6]. Since it was found that organic materials have large nonlinearity and ultrafast response time [4], the research on optical limiting organic materials have become of great importance [5]. Nonlinear absorptive organic dyes are among the most widely studied optical limiting materials [6]. The nonlinear absorption in the dyes is due to reverse saturable absorption (RSA), two-photon absorption (TPA) and saturable absorption (SA) dependent on the change in the absorption (increase or decrease) with increase in intensity.

Both TPA and RSA lead to increase in absorption of the sample with increasing in intensity [7]. For a given organic sample at fixed wavelength, either TPA or RSA is the dominant mechanism leading to increase in absorption with intensity. RSA is observed when excited state absorption (ESA) is greater than the ground state absorption, which results in decrease in the transmission through the medium with increasing in the input intensity. ESA and RSA are the most common mechanisms for the nonlinear optical limiting behavior of organic materials $[8,9]$.

Recently, Geethakrishnan et al. studied the third-order nonlinear optical response of a triphenylmethane dye (acid blue 7) using the $Z$-scan technique with a continuous-wave (CW) $\mathrm{He}-\mathrm{Ne}$ laser radiation at

* corresponding author; e-mail: scientific@aec.org.sy (M.D. Zidan)
$633 \mathrm{~nm}$ [10]. The nonlinear responses and optical limiting performance of a dye type acid, called fast green FCF, are investigated under irradiation of $35 \mathrm{~mW} \mathrm{He}-\mathrm{Ne}$ laser [11]. The nonlinear optical absorption, refraction and optical limiting behavior of an organic dye, neutral red, were investigated under excitation with nanosecond laser pulses at $532 \mathrm{~nm}[7]$.

In this paper, we present the results of our investigations on the optical limiting behavior of organic dye, acid blue 29, under excitation of a low power $\mathrm{CW} \mathrm{He}-\mathrm{Ne}$ laser irradiation. Acid blue 29 is dissolved in ethanol with four concentrations.

\section{Experimental techniques}

The acid blue 29 was purchased from Aldrich and used without any purification. Figure 1 shows the molecular structure of acid blue 29 dye. The UV-visible absorption spectrum of acid blue 29 was recorded using UV-3101 PC Shimadzo Spectrophotometer.<smiles>Nc1c(/N=N/c2cccc([N+](=O)[O-])c2)c(S(=O)(=O)O)cc2cc(S(=O)(=O)[O-])c(/N=N/c3ccccc3)c(O)c12</smiles>

Fig. 1. Molecular structure of acid blue 29 dye.

The acid blue 29 sample at four concentrations of $2 \times 10^{-4}, 4 \times 10^{-4}, 6 \times 10^{-4}$ and $8 \times 10^{-4} \mathrm{M}$ were prepared as ethanol solution. The test cell used in this work was $2 \mathrm{~mm}$ thick quartz cell.

The optical limiting measurements were performed with a plane polarized Gaussian beam CW He-Ne laser 
of $35 \mathrm{~mW}$ output power. The laser beam waist at the focus was measured to be $30 \mu \mathrm{m}$. The maximum applied irradiance on the sample was $2800 \mathrm{~W} \mathrm{~cm}^{-2}$.

The optical geometry used for transmittance measurement is shown in Fig. 2. An attenuator (Glan polarizer) was used to control the incident power on the sample. The laser beam was focused with $10 \mathrm{~cm}$ focal length quartz lens, to increase the beam irradiance at the sample; $35 \mathrm{~mm}$ focal lens was located behind the sample cell to collect the transmitted laser light. Lab-Master Ultima Coherent power meter was used to measure the incident and transmitted power of the laser beam. Laser-cam IIID Coherent beam profiler was used to measure the laser spot diameter behind the sample.

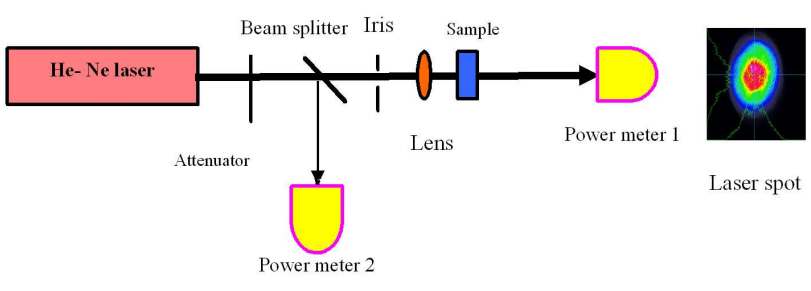

Fig. 2. The optical geometry of the experimental setup.

\section{Results and discussions}

The optical absorption of the acid blue 29 dye in ethanol with $10^{-4} \mathrm{M}$ concentration shows linear absorption peak nearby $630 \mathrm{~nm}$ as shown in Fig. 3.

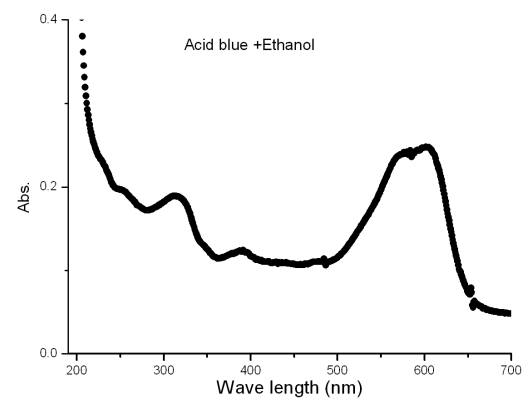

Fig. 3. The UV-vis absorption spectrum of acid blue 29 in ethanol with $10^{-4} \mathrm{M}$ concentration.

We have measured the laser spot size behind the sample at different concentrations by using a Laser-cam IIID Coherent beam profiler. Figure 4 shows images of the laser spot when the sample is placed at the focal of the lens. As seen, the laser spot size increases with increasing dye concentrations, which indicates induced divergence on the beam passing through the sample. In other words, the sample acts as a negative lens when laser beam passes through it.

Moreover, induced self-diffraction patterns are indeed observed at relatively high intensities starting from
$15 \mathrm{~mW}$. Figure 4 shows laser spots taken for samples $2 \times 10^{-4}, 4 \times 10^{-4}, 6 \times 10^{-4}$, and $8 \times 10^{-4} \mathrm{M}$ concentration, respectively, at far-field measurement using a $5 \mathrm{~cm}$ focal lens. As shown the number of fringes is increased with increasing concentration in which a linear relation can be established by plotting the number of fringes as function of concentration, as seen in Fig. 5. It is well known that for Gaussian beam propagation through a lens-like medium, the maximum variation of relative index $\Delta n_{\max }$ is proportional to the number of fringes [12]. Thus one can expect a linear variation of $\Delta n_{\max }$ with the concentration of the acid blue from the measured data. Further investigation of this phenomenon will appear in forthcoming work.
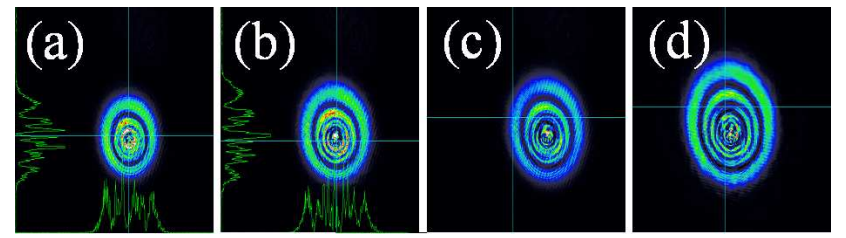

Fig. 4. Laser spot recorded behind the sample at four different concentrations: (a) $2 \times 10^{-4}$, (b) $4 \times 10^{-4}$, (c) $6 \times 10^{-4}$, and (d) $8 \times 10^{-4} \mathrm{M}$.

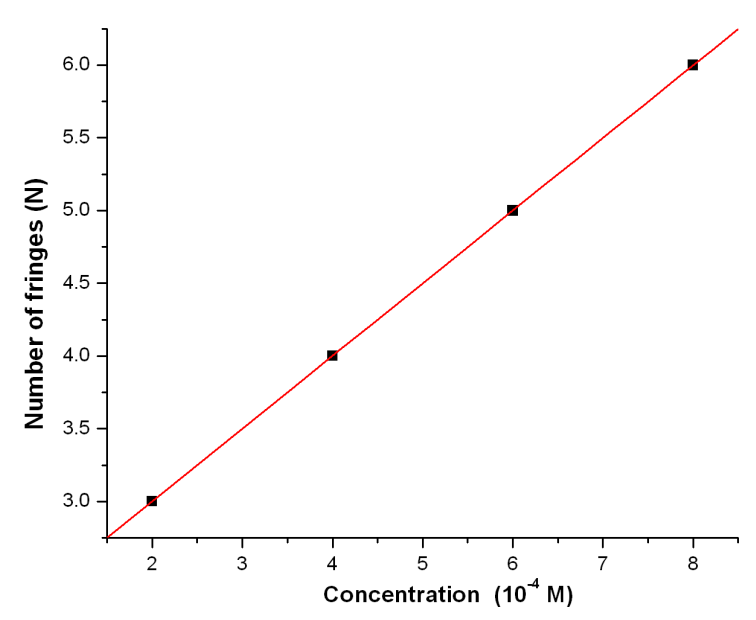

Fig. 5. Number of fringes $(N)$ as function of sample concentrations.

For optical limiting measurements, the sample is located at the focal plane of the lens and the input power of the laser beam is varied. Figure 6 shows the results of the transmission measurements in acid blue 29 dissolved in ethanol samples $2 \times 10^{-4}, 4 \times 10^{-4}, 6 \times 10^{-4}$, and $8 \times 10^{-4} \mathrm{M}$ concentration as a function of incident power, varying from $1 \mathrm{~mW}$ up to $25 \mathrm{~mW}$. It is seen clearly at low incident powers that the output power varies linearly with input power. The linear transmittance obeyed Beer's law: $I=I_{0} \mathrm{e}^{-\alpha L}$, where $I, I_{0}, \alpha$ and $L$ are the incident energy, the output energy, the absorption coefficient and the sample path length, respectively. At high input 
intensity, the linear transmission of $75 \%, 55 \%, 35 \%, 25 \%$ at $2 \times 10^{-4}, 4 \times 10^{-4}, 6 \times 10^{-4}$, and $8 \times 10^{-4} \mathrm{M}$ concentration began deviation from linearity at $23,18,8$, and $6 \mathrm{~mW}$, respectively. One can observe an optical limiting property with clamped output intensity. At high concentration the output power reached a plateau at low input power. The optical limiting responses of the low-concentration solution are generally much weaker than those of more concentrated solutions, while high concentrated solution exhibits strong optical limiting. This indicates that the number density of dye in the laser beam is the main factor affecting the clamped level. From the threshold intensity for optical limiting for each sample, it can be seen that the optical power limiting threshold is inversely proportional to the concentration. Also, the data show that as the concentration increases, a reduction in linear transmittance as well as the clamping level is observed. The observed results confirm previous findings that the concentration effect is remarkable $[6,13]$. Table summarizes the calculated values of linear absorption coefficient, linear transmission $(T)$, and the optical limiting threshold at different four concentrations.

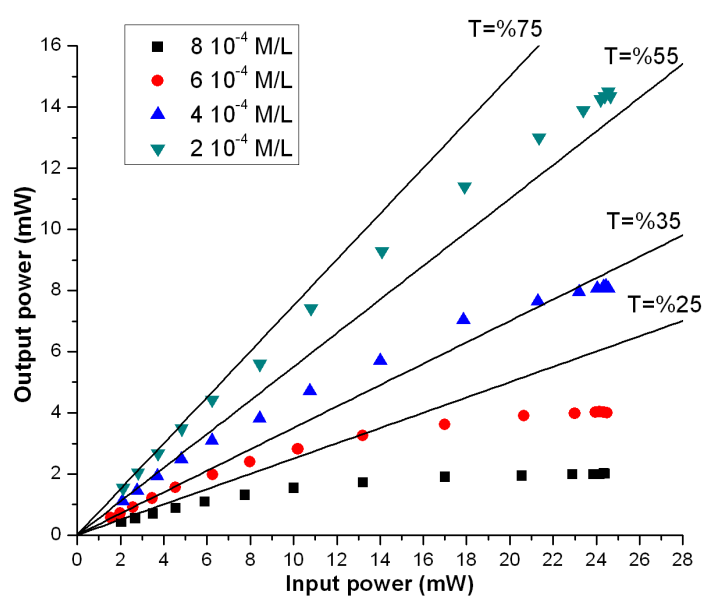

Fig. 6. Variation in output power with input power through acid blue 29 dissolved in ethanol with four different concentrations.

Several mechanisms have been proposed for optical limiting in organic dyes including RSA, nonlinear scattering, multiphoton absorption, thermal defocusing/scattering, nonlinear refraction and induced scattering $[13,14]$. Our optical limiting measurements in acid blue 29 show the participation of both mechanisms: RSA and nonlinear refraction. The RSA contribution is expected from the nonlinear behavior of the measured output power as a function of the input power, in these measurements the total transmitted power through the sample is collected by means of a positive lens of $35 \mathrm{~mm}$ focal length. The discrepancy of the measured transmitted power from the linear behavior observed above $6 \mathrm{~mW}$ in Fig. 6 is related to the nonlinear absorption which in turn originated from the RSA mechanism in the acid
TABLE

Table summarizes the calculated values of linear absorption coefficient, linear transmission $(\% T)$, and $\Delta n$ the optical limiting threshold at four concentrations.

\begin{tabular}{c|c|c|c}
\hline \hline $\begin{array}{c}\text { Sample } \\
\text { concentrations }\end{array}$ & $\begin{array}{c}\text { Linear } \\
\text { absorption } \\
\text { coefficient } \\
\alpha\left[\mathrm{mm}^{-1}\right]\end{array}$ & $\begin{array}{c}\text { Linear } \\
\text { transmission } \\
{[\% \mathrm{~T}]}\end{array}$ & $\begin{array}{c}\text { Power limiting } \\
\text { threshold } \\
W_{\text {th }}[\mathrm{mW}]\end{array}$ \\
\hline $2 \times 10^{-4} \mathrm{M}$ & 0.06 & 75 & 23 \\
$4 \times 10^{-4} \mathrm{M}$ & 0.13 & 55 & 18 \\
$6 \times 10^{-4} \mathrm{M}$ & 0.23 & 35 & 8 \\
$8 \times 10^{-4} \mathrm{M}$ & 0.30 & 25 & 6
\end{tabular}

blue 29. The nonlinear absorption processes of organic dye molecules have been described by a five-energy-level model $[6,15]$, whereas the induced self-diffraction pattern was observed at relatively high powers $(15 \mathrm{~mW})$, which is entirely associated to the variation of the nonlinear refractive index inside the sample. The detailed theoretical analysis of both mentioned mechanisms in the acid blue 29 will be the subject of a forthcoming paper.

However, it has been noticed that other mechanism could be involved at higher intensities like induced scattering that will be investigated later.

Finally, absorption spectra of the sample were acquired before and after the laser irradiation and found that the pattern and its intensity have almost no change, hinting that the prepared sample processes photostability.

\section{Conclusions}

We have presented the measurements of the optical limiting performance, in the acid blue 29 at $2 \times 10^{-4}$, $4 \times 10^{-4}, 6 \times 10^{-4}$, and $8 \times 10^{-4} \mathrm{M}$ concentrations exposed to a low power CW He-Ne laser irradiation at $632.8 \mathrm{~nm}$. Power limiting threshold as low as $6 \mathrm{~mW}$ is measured for solution concentration of $8 \times 10^{-4} \mathrm{M}$. Induced self-diffraction patterns are observed for different solution concentrations at relatively high intensities.

\section{Acknowledgments}

The authors would like to thank Prof. I. Othman, the Director General of AECS for his encouragement and support.

\section{References}

[1] T.J. Bunning, I.V. Natarajan, M.G. Schmitt, B.L. Epling, R.L. Crane, Appl. Opt. 30, 4341 (1991).

[2] L.W. Tutt, A. Kost, Nature 356, 225 (1992).

[3] R. Chari, S.R. Mishra, H.R. Rawat, S.M. Oak, Appl. Phys. B 62, 293 (1996).

[4] H. Wang, J. Xu, C. Yu, Proc. SPIE 2321, 10 (1994). 
[5] T.J. Bunning, L.V. Natamian, Opt. Lett. 15, 700 (1990).

[6] Ya-P. Sun, J.E. Riggs, Int. Rev. Phys. Chem. 18, 43 (1999).

[7] M. George, C.I. Muneer, C.P. Singh, K.S. Bindra, S.M. Oak, Opt. Laser Technol. 40, 373 (2008).

[8] J.E. Riggs, Ya-P. Sun, J. Phys. Chem. A 103, 485 (1999).

[9] S.S. Harilal, C.V. Bindhu, V.P.N. Nampoori, P.G. Vallabhan, J. Appl. Phys. 86, 1388 (1999).

[10] T. Geethakrishnan, P.K. Palanisamy, Opt. Commun. 270, 424 (2007).
[11] K. Jamshidi-Ghaleh, S. Salmani, M.H. Majles Ara, Opt. Commun. 271, 551 (2007).

[12] R. Menzel, Photonics: Linear and Nonlinear Interaction of Laser Light and Matter, 2nd ed., Springer, Berlin 2001.

[13] L.W. Tutt, T.F. Boggess, Prog. Quantum Electron., 17, 299 (1993).

[14] R. Tong, H. Wuc, B. Lia, R. Zhua, G. Youa Qian Sh, Y. Linc, R. Cai, Physica B 366, 192 (2005).

[15] Fang Li, Qiguang Zheng, Guang Yang, Nengli Dai, Peixiang Lu, Mater. Lett. 62, 3059 (2008). 